\title{
Évaluation d'un modèle dynamique de croissance et de développement de la tomate (Lycopersicon esculentum Mill), TOMGRO, pour différents niveaux d'offre et de demande en assimilats
}

\author{
N Bertin, C Gary * \\ INRA, station de bioclimatologie, centre de recherches d'Avignon, BP 91, F84143 Montfavet Cedex, France
}

(Reçu le 5 février 1992, accepté le 1 er février 1993)

\begin{abstract}
Résumé - Le modèle TOMGRO, modèle de croissance et de développement de la tomate, a été évalué pour différents niveaux d'activité des sources et des puits d'assimilats, créés par un enrichissement en $\mathrm{CO}_{2}$ et par la taille des inflorescences. Notre objectif principal étant de tester les hypothèses de répartition des assimilats et la fonction de nouaison des fruits, les paramètres du modèle de photosynthèse ont été ajustés de manière à bien simuler la production journalière de matière sèche. Les principaux paramètres d'entrée pour les sous-modèles de croissance et de développement ont été mesurés expérimentalement. Ainsi ajusté, le modèle simule bien la croissance en surface des feuilles, mais ne prend pas en compte les variations de stocks d'assimilats au niveau des feuilles, et leurs répercussions probables sur la masse foliaire. La croissance totale des fruits mesurée est relativement indépendante du nombre de fleurs par inflorescence. Le modèle simule bien cette croissance mais il sous-estime légèrement la croissance des inflorescences taillées à 3 fleurs. La fonction de nouaison, établie à partir de suivis du développement et de simulations de l'équilibre source/puits, permet une très bonne estimation du nombre d'organes noués. Cela confirme l'hypothèse selon laquelle le taux de nouaison d'une inflorescence dépend du rapport entre les forces des sources et des puits d'assimilats. Ce premier ajustement sur une variété à gros fruits a confirmé la robustesse du modèle mais il a également permis de proposer des améliorations. Les assimilats excédentaires devraient être stockés dans un compartiment pouvant exercer un effet tampon pendant les périodes où l'offre est limitante. La force de puits des appareils reproducteurs et végétatifs devrait être mesurée précisément pour différents types variétaux, et dans différentes conditions climatiques. Enfin il reste à déterminer si les fonctions de répartition des assimilats et de nouaison sont toujours les mêmes dans des conditions très limitantes ou si certains organes deviennent alors prioritaires.
\end{abstract}

Lycopersicon esculentum Mill / force des sources / force des puits / répartition des assimilats / nouaison / modélisation

Summary - Evaluation of TOMGRO, a dynamic model of growth and development of tomato (Lycopersicon esculentum Mill) at various levels of assimilate supply and demand. TOMGRO, a tomato growth and development model, has been examined under different levels of assimilate source and sink activities, induced by $\mathrm{CO}_{2}$ enrichment and truss thinning. The main purpose was the evaluation of the assumptions on dry matter partitioning and fruit setting. The photosynthesis submodel has been calibrated to fit the daily dry-matter production. The main input parameters to the development and growth submodels have been experimentally measured. The calibrated model provides good simulations of the leaf area expansion, but it takes no account of the variations in the assimilates stored in leaf blades. Total fruit growth is well simulated in spite of a small underestimation for of development and simulations of source/sink balance leads to good simulations of the number of set fruits. This result confirms the hypothesis that fruit set depends on the ratio between assimilate source and sink activities. This calibration with a beef tomato cultivar proves the robustness of the model and permits some improvements to be suggested. The surplus assimilates should be stored in a pool, which could exert a buffer effect during low supply periods. Sink strength of reproductive and vegetative parts should be measured for different cultivars, and under various climatic conditions. Finally, whether the functions of assimilate distribution and fruit set are still valid under very low supply conditions or whether some organs have priority over the others remains to be determined. 


\section{INTRODUCTION}

La gestion raisonnée du climat des serres doit s'appuyer sur une bonne connaissance du système serre-peuplement végétal. Au sein de ce système, les relations entre climat et fonctionnement du peuplement végétal doivent être étudiées à plusieurs échelles du temps, qui correspondent à plusieurs niveaux de prise de décision (Baille, Boulard et Gary, 1990). Cela justifie le développement de modèles d'élaboration du rendement des cultures sous serre permettant, à l'échelle d'un cycle de culture, la définition de stratégies globales de conduite du climat et, au jour le jour, la détermination de consignes optimales (en particulier de température et de teneur en $\mathrm{CO}_{2}$ ). Ces modèles doivent permettre d'anticiper les réactions du peuplement végétal à différents climats, en termes de production de biomasse, de production d'organes "capteurs" et de répartition de la biomasse entre ces différents organes.

Les variétés modernes de tomate sont conduites, sous serre, selon un schéma simple de développement. Ce sont généralement des variétés à croissance indéterminée ; elles produisent un étage végétatif (7-8 feuilles), puis une succession d'unités de reproduction comprenant chacune une inflorescence et 3 feuilles. Ces unités, d'âges physiologiques différents, sont en compétition pour les assimilats. Les voies de circulation de ces assimilats ont été déterminées et des relations privilégiées entre les sources et les puits les plus proches ont été mises en évidence (Bonnemain, 1966); en revanche, l'importance et le contrôle des flux sont moins bien connus. Des tentatives récentes de modélisation de l'élaboration du rendement de la tomate font appel à des hypothèses simples, en simulant la répartition des assimilats selon des rapports constants entre organes (Kano et van Bavel, 1988), et/ou selon le rapport entre les forces des différents puits (Heuvelink et Marcelis, 1989; Jones et al, 1991). On entend ici par force de puits la vitesse de croissance potentielle de l'organe considéré, observée quand son approvisionnement en assimilats n'est pas limitant. Cependant, le problème de la nouaison n'est pas pris en compte dans ces modèles, alors qu'il peut sérieusement limiter le nombre de fruits et, par conséquent le rendement, en période hivernale. La nouaison est une étape du développement du fruit qui correspond au début de son grossissement. II y a défaut de nouaison lorsque, après la floraison, un jeune fruit ne grossit pas. Ce phénomène peut être lié à des défauts de fécondation dont les causes (températures et hygrométries extrêmes) ont été identifiées (Maisonneuve et Philouze, 1982). Cependant, lorsque ces obstacles climatiques sont levés pour assurer une fécondation correcte, des défauts de nouaison persistent, particulièrement en période hivernale à faibles niveaux de rayonnement, lorsque 5 à 6 inflorescences sont simultanément en croissance sur la plante et que la compétition pour les assimilats augmente (Picken, 1984; Atherton et Harris, 1986; Wolf et Rudich, 1988). TOMGRO, un modèle de croissance et de développement de la tomate (Jones et al, 1991), fait du rapport entre l'offre (photosynthèse) et la demande (force des différents puits) en assimilats l'élément central à partir duquel sont simulés la répartition de la matière sèche et les défauts de nouaison. Les défauts de nouaison résulteraient d'un déséquilibre entre l'activité des sources et la demande des puits d'assimilats. Cependant cette dernière partie du modèle n'avait pas, jusqu'à présent, été confrontée à des données expérimentales.

Notre objectif est de présenter ici une analyse critique du modèle TOMGRO. Un travail expérimental a permis de créer différents niveaux d'activité des sources (enrichissement en $\mathrm{CO}_{2}$ ) et des puits (taille des inflorescences), de façon à apprécier l'intérêt de la démarche des auteurs des auteurs du modèle TOMGRO et à proposer des améliorations. En particulier, les résultats permettent de discuter l'intérêt d'introduire un compartiment de stockage des assimilats pouvant exercer un rôle tampon entre l'offre des sources et la demande des puits, ainsi qu'une hiérarchie entre les différents puits selon leur position sur la plante.

\section{PRÉSENTATION DU MODĖLE TOMGRO}

\section{Présentation générale}

Le modèle TOMGRO décrit la plante à travers 7 variables d'état : les nombres et poids de matière sèche des entre-nœuds, des feuilles et des fruits, et la surface foliaire. Ces 7 variables sont des vecteurs comprenant $N$ classes d'âge physiologique. Tout organe entre dans la première classe d'âge quand il est initié et atteint la classe $N$ quand sa croissance est terminée. La vitesse de passage d'une classe à l'autre dépend du 
nombre total de classes, de la température et de la teneur en $\mathrm{CO}_{2}$. Le modèle fonctionne selon 2 pas de temps: (a) l'heure, au cours de laquelle sont calculées les vitesses d'apparition et de vieillissement des différents organes, la photosynthèse brute et la respiration d'entretien; et (b) la journée, au cours de laquelle sont intégrées les différentes fonctions de croissance et de développement, ce qui permet une remise à jour des 7 variables d'état. L'ensemble des sousmodèles contribuent au calcul journalier du rapport entre l'offre et la demande en assimilats, appelés respectivement "force de la source" et «force des puits» (fig 1). C'est ce rapport qui détermine la croissance réelle des différents organes et le nombre de fruits noués à l'issue de chaque journée.

\section{Production journalière de matière sèche}

La production de matière sèche correspond à la différence entre photosynthèse brute $\left(P_{b^{\prime}}\right.$ en $\mathrm{g}$ $\left.\mathrm{CH}_{2} \mathrm{O} \cdot \mathrm{m}^{-2} . \mathrm{j}^{-1}\right)$ et respiration d'entretien $\left(R_{e}\right)$, multipliée par l'efficience de conversion des assimilats en matière sèche $\left(Y_{G^{\prime}}\right.$ en $\mathrm{g} \mathrm{MS}$. $\left.\left(\mathrm{g} \mathrm{CH}_{2} \mathrm{O}\right)^{-1}\right)$. La photosynthèse brute est calculée heure par heure à l'aide du modèle d'Acock (Acock et al,
1978) dans lequel on fait I'hypothèse que toutes les feuilles présentent la même courbe de réponse de la photosynthèse au rayonnement visible ( () et que le couvert végétal est homogène. L'asymptote $\left(P_{\text {max }}\right)$ de cette courbe de réponse au rayonnement augmente linéairement avec la teneur en $\mathrm{CO}_{2}(C)$ et décroît pour les températures $(T)$ inférieures à $12{ }^{\circ} \mathrm{C}$ ou supérieures à $28^{\circ} \mathrm{C}$.

$$
\begin{aligned}
P_{b}= & \left(P_{\max } / k\right) \cdot \log \left(\left((1-m) \cdot P_{\max }+Q_{e} \cdot k \cdot I\right)\right. \\
& /\left((1-m) \cdot P_{\max }+Q_{e} \cdot k \cdot I \cdot e^{-k \cdot L)}\right)
\end{aligned}
$$

où $k$ est le coefficient d'extinction du rayonnement dans le couvert végétal, $m$ le coefficient de transmission des feuilles, $Q_{e}$ l'efficience quantique de la photosynthèse $\left(\mathrm{mol} \mathrm{CO}_{2}\right.$. (mol photon $^{-1}$ ) et $L$ l'indice foliaire;

$$
P_{\max }=\tau . C . f(T)
$$

où $\tau$ est l'efficience d'utilisation du $\mathrm{CO}_{2}$ ( $\mu \mathrm{mol}$ $\mathrm{CO}_{2} \cdot \mathrm{m}^{-2} \cdot \mathrm{s}^{-1}$ par ppm $\mathrm{CO}_{2}$ ). L'acclimatation aux conditions climatiques (rayonnement ou concentration en $\mathrm{CO}_{2}$ ) et l'âge des feuilles ne sont pas pris en compte. La respiration d'entretien dépend des quantités de matière sèche des organes végétatifs et reproducteurs et de leur

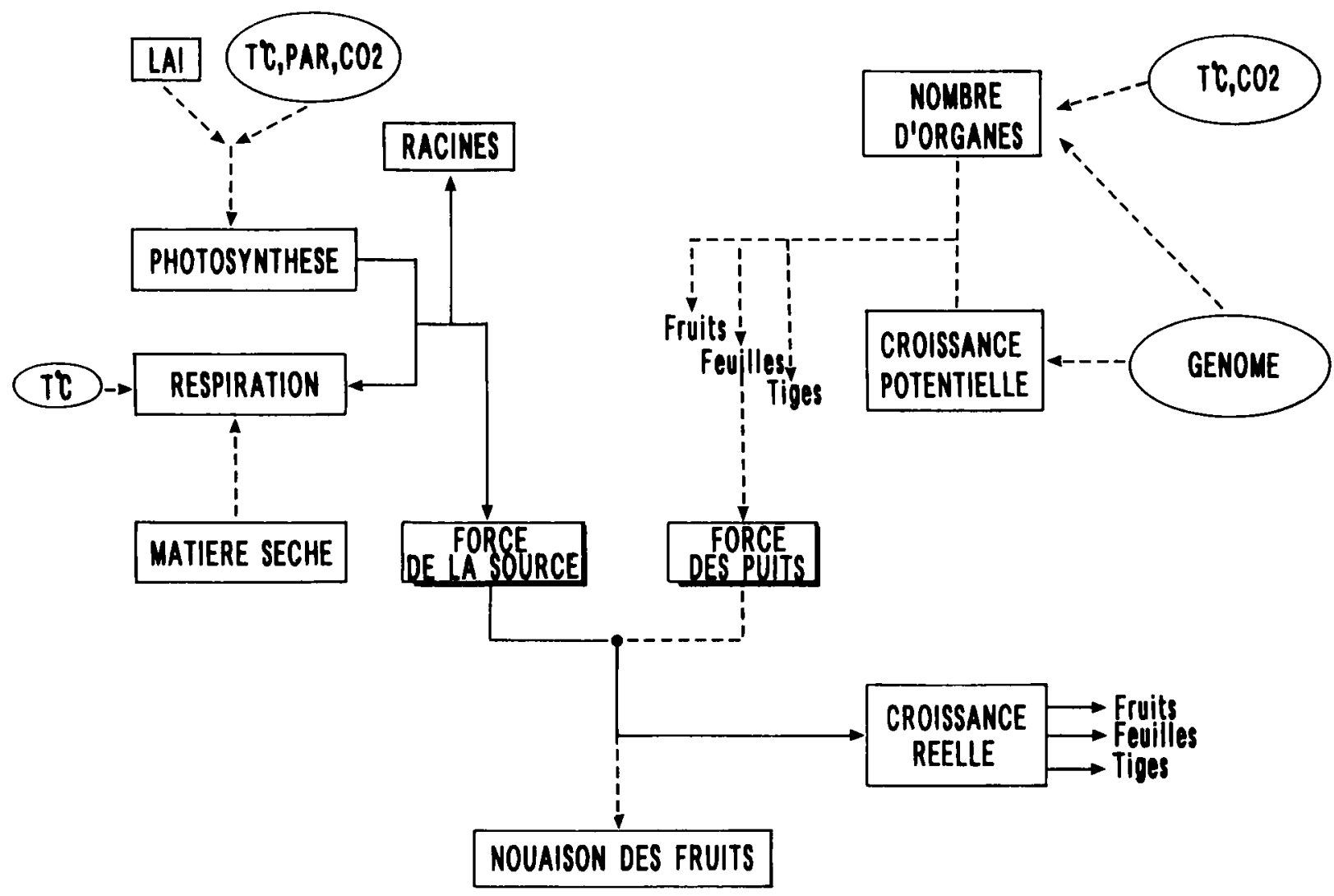

Fig 1. Schéma de fonctionnement du modèle TOMGRO. Flux de matière : $\rightarrow$ et flux d'information : -->- 
coût d'entretien, et elle suit une loi exponentielle de la température. Photosynthèse brute et respiration d'entretien sont intégrées sur $24 \mathrm{~h}$ et converties en $\mathrm{g} \mathrm{CH}_{2} \mathrm{O} \cdot \mathrm{m}^{-2}$.j- $\mathrm{j}^{-1} \mu \mathrm{mol} \mathrm{CO}_{2}$. $\mathrm{m}^{-2} \cdot \mathrm{s}^{-1}$ équivaut à 2, $\left.593 \mathrm{~g} \mathrm{CH}_{2} \mathrm{O} \cdot \mathrm{m}^{-2} \cdot \mathrm{j}^{-1}\right)$. Une partie de la croissance journalière ainsi calculée est directement attribuée aux racines selon une fraction $\left(F_{r t}\right)$ variable avec le stade de développement de la plante (de 0,20 au début du cycle à 0 en fin de cycle). Le reste constitue l'offre journalière de matière sèche ( $\mathrm{O}$ en $\mathrm{g} \mathrm{MS} \cdot \mathrm{m}^{-2} . \mathrm{j}^{-1}$ ), qui est distribuée entre les feuilles, les tiges et les fruits :

$$
O=Y_{G} \cdot\left(P_{b}-R_{e}\right) \cdot\left(1-F_{n}\right)
$$

\section{Organogénèse}

La vitesse d'apparition des nouveaux nœuds (feuilles ou inflorescences), $G_{n d}$, est susceptible de variations génétiques et augmente avec la température et la teneur en $\mathrm{CO}_{2}$. Elle détermine la vitesse d'apparition des feuilles et des inflorescence, la valeur du rapport entre ces 2 types d'organes, $F_{i f}$, étant proche de 0,33. Après la nouaison du premier fruit, la vitesse d'apparition des fruits, $G_{f r}$ est liée avec la vitesse d'apparition des nœuds suivant un rapport, $F_{f n}=G_{f r} / G_{n d}$, qui est fonction lui-même du stade de développement de la plante exprimé en nombre de nœuds déjà apparus.

Le modèle calcule ensuite la fraction de fruits noués, c'est-à-dire d'ovaires fécondés qui démarrent normalement leur croissance. Le coefficient de nouaison $\left(F_{n o}\right)$, compris entre 0 et 1 , suit une fonction linéaire du rapport entre l'offre et la demande en assimilats de l'ensemble des organes en croissance.

\section{Force des puits et répartition de la matière sèche}

La force de puits (ou demande en matière sèche) des feuilles, des tiges et des fruits est définie, pour chaque classe d'âge, par la vitesse de croissance potentielle des organes. Elle présente une forte variabilité génétique. Pour les limbes foliaires, le modèle utilise une vitesse de croissance potentielle en surface, qui est transformée en une demande en matière sèche par l'intermédiaire de la surface spécifique (SLA = Specific Leaf Area). Le SLA de la nouvelle bio- masse foliaire produite chaque jour dépend des conditions climatiques du même jour : il diminue avec la somme de rayonnement et la teneur en $\mathrm{CO}_{2}$ moyenne, et augmente avec la température moyenne. La demande des pétioles est une fraction constante $(0,49)$ de la demande des limbes foliaires, mais elle peut varier avec le stade de développement de la feuille. De même, la demande des entre-nœuds est une fraction de la demande des feuilles entières (limbes plus pétioles), qui varie en fonction du stade de développement de l'organe. Pour chaque type d'organe (feuilles, fruits ou tiges), la demande totale est la somme des forces de puits sur toutes les classes d'âge. Enfin la demande totale des parties aériennes $\left(D\right.$, en $\left.\mathrm{g} \mathrm{MS} . \mathrm{m}^{-2} . \mathrm{j}^{-1}\right)$ est la somme des forces de puits des feuilles $\left(D_{f e}\right)$, des tiges $\left(D_{t i}\right)$ et des fruits $\left(D_{f r}\right)$ :

$$
D=D_{f e}+D_{t i}+D_{f r}
$$

Si le rapport entre l'offre totale $(O)$ et la demande totale $(D)$ est supérieur ou égal à 1 , la croissance potentielle de chaque organe est satisfaite. Lorsque l'offre est supérieure à la demande totale, le modèle ramène l'offre au niveau de la demande : il n'existe pas de compartiment de stockage des assimilats dans la version actuelle du modèle. Si le rapport $O / D$ est inférieur à 1 , la croissance réelle (respectivement $C_{f e^{\prime}} C_{t t^{\prime}}$ et $C_{f r}$ ) se fait au prorata de la demande respective de chaque type d'organe:

$$
\begin{aligned}
& C_{f e}=D_{f e \cdot}(O / D) \\
& C_{f r}=D_{f r}(O / D) \\
& C_{t i}=D_{t i \cdot}(O / D)
\end{aligned}
$$

Pour chaque catégorie d'organes, la croissance en matière sèche est répartie suivant les mêmes règles entre les $N$ classes d'âge physiologique. Par exemple pour la classe de feuilles (i) :

$$
C_{f e}(i)=D_{f e}(i) \cdot(O / D)
$$

\section{MATÉRIEL ET MÉTHODES}

L'objectif de l'expérimentation était de créer différents niveaux d'activité des sources et des puits d'assimilats, afin de tester les hypothèses de nouaison et de répartition de la matière sèche du modèle TOMGRO. 


\section{Conditions de culture}

Les graines de tomates (Lycopersicon esculentum Mill, variété "Capello") ont été semées à $25^{\circ} \mathrm{C}$ dans des cylindres de sable le 24 octobre 1989. Les plants ont été repiqués dans des cubes de laine de roche le 3 novembre 1989 puis plantés le 4 décembre 1989 sous 2 tunnels en polycarbonate à double paroi, à une densité de 2,5 plantes par $\mathrm{m}^{2}$. Les consignes de température jour/nuit étaient fixées à $19 / 16^{\circ} \mathrm{C}$, sans éclairage artificiel. Les plantes ont été conduites de manière classique et étêtées au-dessus de la $12^{\mathrm{e}}$ inflorescence. Les fleurs ont été vibrées mécaniquement 3 fois par semaine afin de limiter les défauts de fécondation en assurant une bonne pollinisation.

\section{Traitements}

Afin de modifier l'activité des sources d'assimilats, l'atmosphère a été enrichie en $\mathrm{CO}_{2}$ dans l'une des 2 serres, à une concentration de 1000 ppm de 8 h 00 à $12 \mathrm{~h} 00$, dès l'apparition de la première inflorescence (20 décembre 1989). À partir du mois de mars (nouajson de la $7^{\mathrm{e}}$ inflorescence), la durée d'enrichissement a été involontairement réduite de moitié à cause des ventilations fréquentes de la serre au printemps. Dès la fin de ce même mois, l'enrichissement ne durait plus que $1 \mathrm{~h} . \mathrm{j}^{-1}$, puis il a été interrompu en avril (nouaison de la 9 inflorescence). L'autre serre n'a recu aucun enrichissement en $\mathrm{CO}_{2}$. Dans chaque serre, le nombre de fruits était maîtrisé par la taille des inflorescences à 3 ou 7 fleurs. La taille a été réalisée au moment de la floraison afin de réduire très tôt la compétition au sein des inflorescences. Nous avonc donc 4 traitements identifiés de la façon suivante:

$T_{7}$ : taille à 7 fleurs sans $\mathrm{CO}_{2}$;

$\mathrm{T}_{3}$ : taille à 3 fleurs sans $\mathrm{CO}_{2}$;

$\mathrm{T}_{7} \mathrm{C}$ : taille à 7 fleurs avec $\mathrm{CO}_{2}$;

$\mathrm{T}_{3} \mathrm{C}$ : taille à 3 fleurs avec $\mathrm{CO}_{2}$.

\section{Observations et mesures}

Le développement des plantes a été suivi 2 fois par semaine, sur 26 plantes de chacun des 4 traitements. Au cours des observations on a noté les nombres de feuilles, d'inflorescences, de boutons floraux, de fleurs épanouies et de fruits noués sur les 10 premières inflorescences.

Une analyse de croissance a été réalisée pour chaque traitement sur des échantillons de 10 plantes, prélevés aux stades plantation puis nouaison des $3^{\mathrm{e}}, 5^{\mathrm{e}}$, $7^{e}$ et $9 e$ inflorescences. La surface foliaire des plantes a été mesurée au planimètre optique ( $\mathrm{Li}$-Cor 3000 ) ; les matières sèches des feuilles, des tiges et des fruits ont été mesurées après un séchage de $72 \mathrm{~h}$ à $80^{\circ} \mathrm{C}$ dans une étuve ventilée.
Les croissances potentielles des fruits et des limbes foliaires ont été mesurées dans le cadre d'un autre essai conduit en 1991 sur la même variété. En théorie, on ne peut observer une croissance potentielle que lorsqu'aucun facteur de croissance (climatique ou minéral) n'est limitant, ce qui est toujours très difficile à vérifier. En réalité, on définit un potentiel pour des conditions climatiques données. La croissance potentielle en matière sèche des fruits a été mesurée sur des plantes à un seul fruit par inflorescence, plantées sur solution nutritive le 26 novembre à une densité de 2,2 plantes par $\mathrm{m}^{2}$. La croissance potentielle en surface des feuilles a été mesurée sur des plantes sans fruits de la même culture. Les courbes de croissance en matière sèche ont été ajustées par une fonction de Gompertz à 3 paramètres. Les plus fortes croissances observées (premier fruit de la $4^{\mathrm{e}}$ inflorescence et feuille au-dessus de cette inflorescence) ont été utilisées pour estimer le potentiel de croissance en matière sèche des fruits et en surface des feuilles.

\section{Analyse critique du modèle}

Notre attention s'est portée dans ce travail sur l'utilisation, dans TOMGRO, du rapport offre sur demande en assimilats comme critère de répartition de la matière sèche et de contrôle du taux de nouaison. II fallait pour cela s'assurer au préalable de la précision de l'estimation par le modèle de la force des sources et des puits d'assimilats. C'est pourquoi, après confrontation des données mesurées et calculées, nous avons procédé à un nouveau calage des paramètres des sous-modèles de photosynthèse et d'organogénèse à partir de nos données expérimentales. En revanche, le calcul des forces de puits est fait à partir de données expérimentales indépendantes et la critique du modèle porte sur la répartition de la matière sèche et sur la nouaison. Tous les résultats expérimentaux sont présentés avec un intervalle de confiance au seuil de $10 \%$.

\section{RÉSULTATS}

\section{Estimation de l'offre en assimilats}

Dans la serre enrichie en $\mathrm{CO}_{2}$, il y a eu une augmentation significative de la production de matière sèche au cours de la première phase de culture ( $2^{\mathrm{e}}$ prélèvement), puis les différences se sont réduites (fig 2). A partir de ce stade, la durée d'enrichissement était limitée par les besoins de ventilation de la serre et, contrairement à l'objectif du traitement, l'offre en assimilats était comparable dans les 2 compartiments de serre. À la fin de la période d'observation (nouai- 

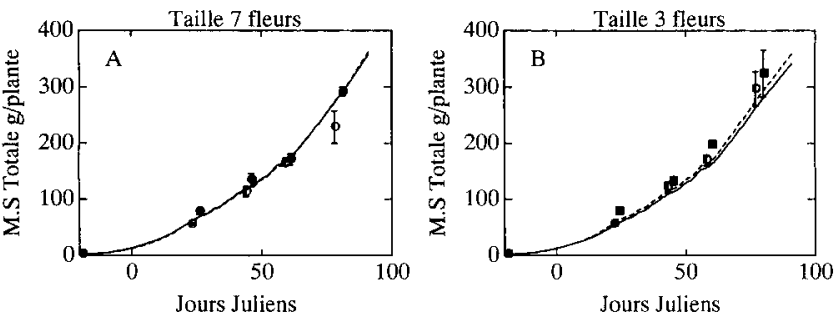

Fig 2. Croissance en matière sèche des parties aériennes. (A) taille à 7 fleurs $(B)$ taille à 3 fleurs. Données expérimentales (moyenne sur 10 plantes) : T7 (O) T7C (@) T3 ( $\square)$ T3C $(\square)$ et données simulées par le modèle : T7 et T3 $(-)$, T7C et T3C (---)

son de la 9 inflorescence), la quantité totale de matière sèche accumulée dans les parties aériennes était significativement inférieure pour le traitement $T_{7}$.

Les paramètres du modèle de photosynthèse de la version originale de TOMGRO (équations [1] et [2]) conduisent à une sous-estimation de la production de matière sèche mesurée au cours de notre expérimentation. Trois de ces paramètres ont donc été réajustés à partir de nos 4 jeux de données expérimentales de manière à simuler au mieux la croissance journalière. $\mathrm{Ce}$ calage a été fait sur la base de valeurs établies au préalable sur un matériel végétal identique (Tchamitchian et Longuenesse, comm pers). Le nouveau jeu de paramètres est le suivant :

$k=0,80$;

$m=0,10$;

$Q_{e}=0,080 \mu \mathrm{mol} \mathrm{CO} 2$. (mol photons) $)^{-1}$;

$\tau=0,10 \mu \mathrm{mol}$ de $\mathrm{CO}_{2} \cdot \mathrm{m}^{-2} \cdot \mathrm{s}^{-1}$ par ppm $\mathrm{CO}_{2}$;

$Y_{G}=0,75 \mathrm{~g} \mathrm{MS} \cdot\left(\mathrm{gCH}_{2} \mathrm{O}\right)^{-1}$.

La respiration d'entretien est calculée sur la base des coefficients d'entretien utilisés dans la version originale du modèle : $0,015 \mathrm{gCH}_{2} \mathrm{O} .(\mathrm{g}$ $M S)^{-1} .^{-1}$ pour les parties végétatives et $0,010 \mathrm{~g}$ $\mathrm{CH}_{2} \mathrm{O} .(\mathrm{g} \mathrm{MS})^{-1} \mathrm{j}^{-1}$ pour les fruits à $20^{\circ} \mathrm{C}$. L'effet de la température est pris en compte grâce à un facteur $Q_{10}$ ramené de 2,0 à 1,4 (Gary, 1988). La surface foliaire simulée par le modèle TOMGRO est très proche de la surface mesurée (fig 3 ) et le sous-modèle de photosynthèse utilise directement l'indice foliaire calculé chaque jour par le sous-modèle de croissance.

Après le calage du sous-modèle de photosynthèse, la croissance en matière sèche simulée
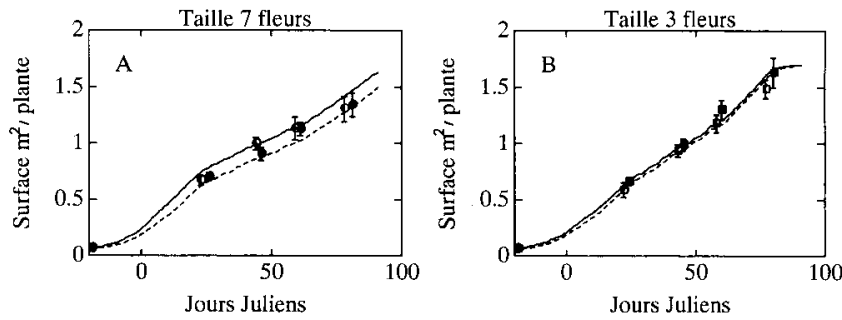

Fig 3. Croissance en surface des feuilles. (A) taille à 7 fleurs (B) taille à 3 fleurs. Données expérimentales (moyenne sur 10 plantes) : T7 (O) T7C (๑) T3 $(\square)$ T3C ( $\mathbf{\square}$ ) et données simulées par le modèle : T7 et T3 $(-)$, T7C et T3C $(--)$.

par le modèle (fig 2) est relativement indépendante de l'enrichissement en $\mathrm{CO}_{2}$ mais, pour les 4 traitements, la simulation est satisfaisante, malgré une légère sous-estimation pour les plantes taillées à 3 fleurs par inflorescence.

\section{Estimation de la demande en assimilats}

Trois des paramètres utilisés pour la simulation de l'organogenèse ont été ajustés à partir des observations de développement faites sur les 4 traitements :

$G_{n d}=0,432$ nœud/jour sans enrichissement en $\mathrm{CO}_{2}$, et

$G_{n d}=0,396$ nœud/jour avec enrichissement en $\mathrm{CO}_{2}$

$F_{f n}=1,69$ fruit/nœud initié sur les inflorescences à 7 fleurs, et

$F_{f n}=0,83$ fruit/nœud initié sur les inflorescences à 3 fleurs ;

$F_{\text {if }}=0,34$ pour les 4 traitements.

L'enrichissement en $\mathrm{CO}_{2}$ a légèrement ralenti le développement de la plante, contrairement à ce qu'avaient observé les auteurs de TOMGRO. Dans le sous-modèle développement, nous avons donc éliminé l'effet positif du $\mathrm{CO}_{2}$ sur la vitesse d'initiation des organes. Ainsi ajusté, le modèle simule très précisément le nombre total de puits sur la plante : feuilles, inflorescences ou fruits.

Les demandes en assimilats des feuilles et des fruits, mesurées sur la variété à gros calibre qui a été retenue, sont supérieures aux valeurs initiales du modèle (Jones et al, 1991). La vi- 
tesse de croissance maximale des fruits (c'est-àdire la pente au point d'inflexion déterminée par la loi de Gompertz) est de 1,03 g.(fruit) ${ }^{-1} . \mathrm{j}^{-1}$, et elle est atteinte avant que le fruit n'ait réalisé la moitié de son développement. Cette valeur était de $0,27 \mathrm{~g}$.(fruit) ${ }^{-1} . \mathrm{j}^{-1}$ dans la version originale du modèle, et de $0,20 \mathrm{~g}$.(fruit) $)^{-1}$. $^{-1}$ dans le modèle présenté par Heuvelink et Marcelis (1989). Pour les feuilles, la vitesse maximale de croissance en surface est de $51 \mathrm{~cm}^{2}$.(feuille) $)^{-1} . j^{-1}$, celle de la version originale du modèle TOMGRO étant de $29 \mathrm{~cm}^{2}$. (feuille) $)^{-1} \cdot \mathrm{j}^{-1}$.

La demande des tiges est une fraction de la demande des feuilles entières (limbes et pétioles). Cette valeur correspond au rapport de matière sèche entre l'ensemble des feuilles et l'ensemble des tiges sur des plantes en production. La valeur intiale du modèle $(0,49)$ conduit à une forte surestimation de la croissance des tiges réellement mesurée pour les 4 traitements. Pour une simulation correcte, nous avons dû adopter un rapport plus faible et variable avec le stade de développement de la feuille : de 0,45 pour les jeunes organes à 0 pour les organes âgés.

\section{Croissance réelle et nouaison des fruits}

La surface foliaire est peu sensible aux traitements (fig 3), excepté à la fin de la période d'observation où la surface mesurée est supérieure sur les plantes à 3 fleurs par inflorescence. Le modèle simule bien ces différences.

La croissance en matière sèche des feuilles est plus sensible aux traitements appliqués (fig 4). Dans la serre enrichie en $\mathrm{CO}_{2}$, on peut observer une forte accumulation de matière sèche dans les feuilles dès le deuxième prélèvement et ce, quelle que soit la charge en fruits. À partir du $3^{e}$ prélèvement, l'effet de l'enrichissement en $\mathrm{CO}_{2}$ a disparu pour les plantes taillées à 7 fleurs, mais il reste significatif pour les plantes taillées à 3 fleurs. Le modèle simule une croissance différente pour les 2 niveaux de $\mathrm{CO}_{2}$, mais il ne simule pas les variations observées lors du deuxième prélèvement (fig 4).

La croissance en matière sèche des fruits n'est pas significativement différente entre les 4 traitements, excepté lors du dernier prélèvement où la croissance est inférieure pour le traitement $\mathrm{T}_{7}$ (fig 5). Le modèle simule une croissance identique pour les 2 niveaux de $\mathrm{CO}_{2}$ et il surestime donc la croissance finale des fruits du traitement
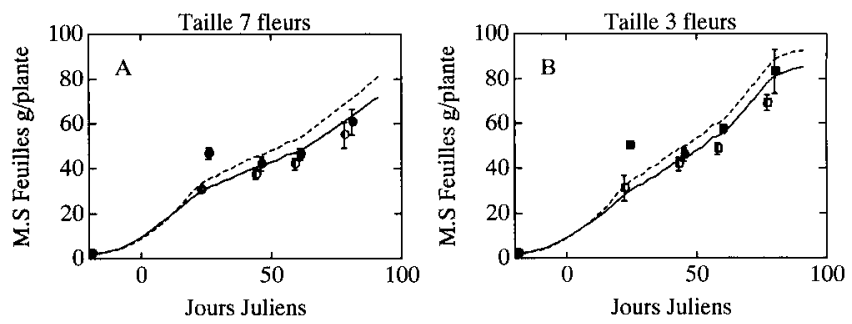

Fig 4. Croissance en matière sèche des feuilles. (A) taille à 7 fleurs (B) taille à 3 fleurs. Données expérimentales (moyenne sur 10 plantes) : T7 (O) T7C (O) T3 ( $\square$ ) T3C (口) et données simulées par le modèle : T7 et T3 (-), T7C et T3C (--) .
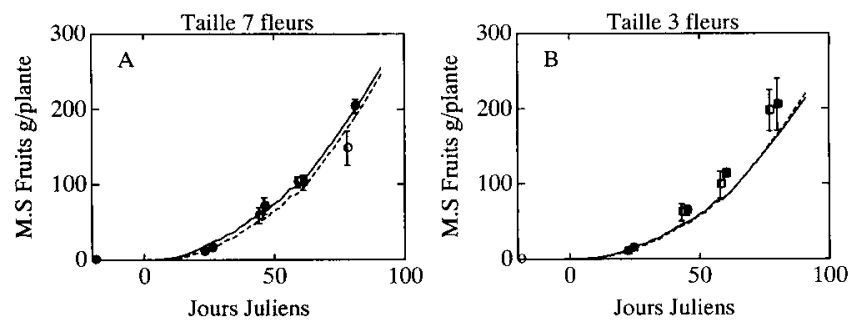

Fig 5. Croissance en matière sèche des fruits. (A) taille à 7 fleurs (B) taille à 3 fleurs. Données expérimentales (moyenne sur 10 plantes) : T7 (O) T7C (O) T3 (口) T3C (回) et données simulées par le modèle : T7 et T3 (-), T7C et T3C (--) .

$T_{7}$. Pour les plantes taillées à 3 fleurs, le modèle sous-estime en permanence la croissance des fruits (fig 5), de la même façon qu'il sousestimait légèrement la croissance totale en matière sèche pour ces 2 traitements (fig 2).

L'évolution de la répartition de la matière sèche entre feuilles, tiges et fruits varie peu d'un traitement à l'autre (fig 6). L'enrichissement en $\mathrm{CO}_{2}$ en début de cycle se traduit par une augmentation de la fraction de matière sèche affectée aux feuilles lors du deuxième prélèvement,
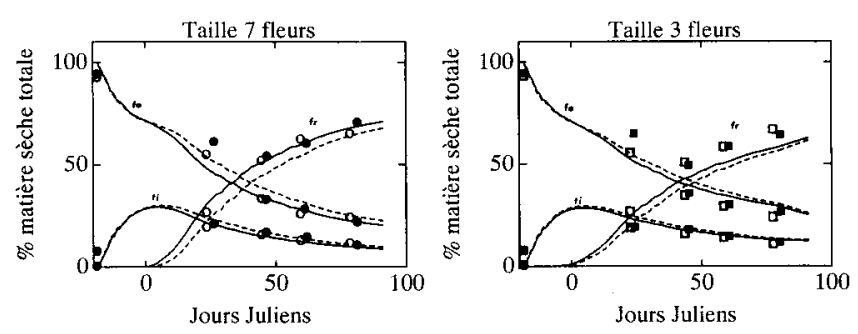

Fig 6. Répartition de la matière sèche aérienne entre les feuilles, les tiges et les fruits. (A) taille à 7 fleurs $(B)$ taille à 3 fleurs. Données expérimentales : T7 (O) T7C (O) T3 ( $\square$ ) T3C (a) et données simulées par le modèle : T7 et T3 (-), T7C et T3C (---). 
ce que prévoit effectivement le modèle. Par contre, le nombre de fruits par inflorescence n'a pas d'effet sur la répartition de la matière sèche, alors que le modèle simule une répartition des assimilats différente selon la taille des inflorescences.

La fonction de nouaison (fig 7) a été établie à partir des comptages d'organes d'une part et du rapport offre/demande calculé par le modèle d'autre part. Nous avons retenu une valeur moyenne de ce rapport sur 3 j parce que la nouaison est une événement difficile à dater au jour près et que les réserves glucidiques peuvent, sur une courte période, limiter les effets d'une offre limitante (Gary et al, 1993). Lorsque le rapport offre/demande descend au-dessous de 0,35 , une fraction des nouveaux fruits apparus ne noue pas (leur diamètre reste inférieur à $2 \mathrm{~cm}$, malgré une bonne fécondation de la fleur). Cette fraction augmente quand le rapport offre/ demande diminue, suivant la relation :

$$
F_{n o}=0,80-2,28 \cdot O / D
$$

II est difficile de savoir exactement ce qui se passe quand ce rapport devient $<0,1$ étant donné l'absence de points expérimentaux dans cette zone. Lorsque le modèle simule bien la croissance en matière sèche des fruits (taille à 7 fleurs par inflorescence), on peut supposer que le rapport offre/demande des fruits est estimé correctement. Dans ce cas, le modèle simule très précisément le nombre de fruits noués (fig 8). Pour la taille à 3 fleurs par inflorescence (traitements $T_{3}$ et $T_{3} C$ ) la nouaison est sous-estimée car l'offre en assimilats pour les fruits, et donc le rapport offre/demande, sont eux-mêmes sousestimés (figs 5 et 6 ).

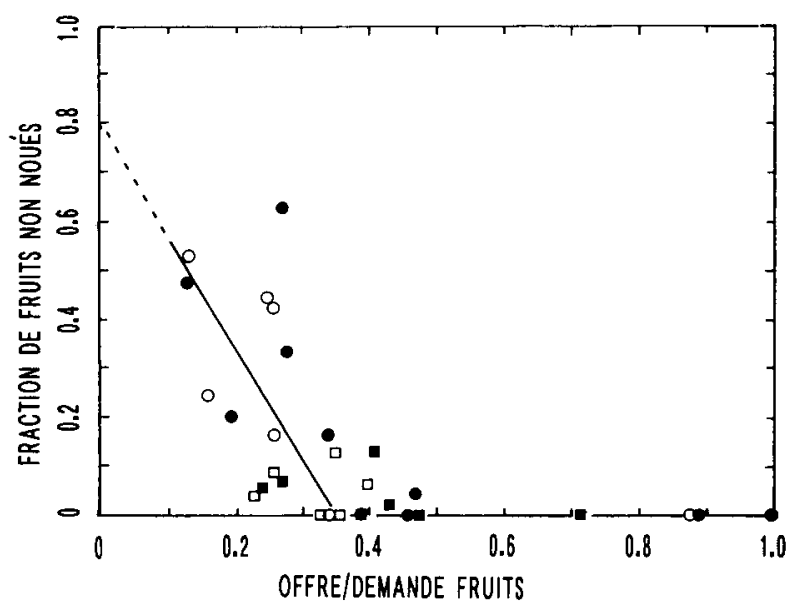

Fig 7. Fonction de nouaison. Données expérimentales (T7 : O; T7C : ; T3: $\square ;$ T3C : $\mathbf{\square})$ et fonction ajustee $(-)$.

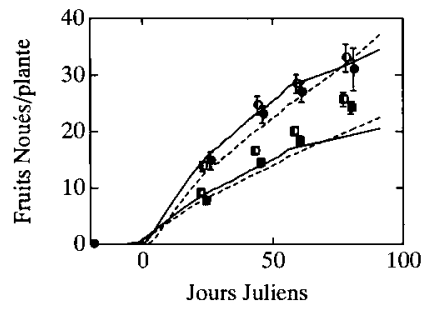

Fig 8. Nombre total d'organes noués par plante. Données expérimentales (moyenne sur 10 plantes) : T7 (O) T7C (๑) T3 $(\square)$ T3C (口) et données simulées par le modèle : T7 et T3 $(-)$ T7C et T3C (---).

\section{DISCUSSION ET CONCLUSION}

Le calage du sous-modèle de photosynthèse nous a conduits à retenir une efficience quantique $\left(Q_{e}\right)$ élevée par rapport aux valeurs que l'on peut trouver dans la littérature (Acock et al, 1978; Spitters et al, 1991 ; Tchamitchian et Longuenesse, 1991). La modification de certains paramètres du modèle était le moyen le plus simple d'obtenir une simulation correcte de l'offre quotidienne en assimilats. Mais on aurait également pu discuter la forme même de ce modèle. Sa simplicité ne lui permet pas de prendre en compte certaines variables comme le rapport diffus/direct du rayonnement incident. Selon la hauteur du soleil, la composante diffuse pénètre plus profondément dans le couvert végétal, permettant ainsi une meilleure répartition du rayonnement entre les différents étages foliaires. Or les caractéristiques de la couverture de la serre utilisée (polycarbonate double paroi) sont telles que le rayonnement incident était essentiellement diffus. Cela a pu contribuer à augmenter la photosynthèse du couvert (Tchamitchian et Longuenesse, 1993). La sous-estimation par le modèle de la croissance totale des plantes taillées à 3 fleurs par inflorescence peut résulter d'une surestimation de la respiration et/ou d'une sousestimation de la photosynthèse. L'effet éventuel du nombre et de la taille des puits sur les paramètres de ces 2 fonctions est difficile à établir. En effet, le sous-modèle de respiration prend en compte le nombre et la taille des puits présents. Par ailleurs, c'est dans les traitements $T_{3}$ et $T_{3} C$, dans lesquels on a limité le nombre de puits, qu'on aurait pu s'attendre à un effet rétroactif négatif d'une accumulation d'assimilats sur la photosynthèse et donc à une moindre croissance en matière sèche totale. Cependant chez la tomate, cet effet rétroactif sur la photosynthèse n'a pas été clairement mis en évidence (Ho et al, 1983). 
Lors du dernier prélèvement (nouaison de la ge inflorescence), la surface foliaire mesurée est significativement inférieure pour les plantes taillées à 7 fleurs. Le niveau de compétition entre les organes aurait donc un léger effet sur la croissance en surface des feuilles. L'augmentation de la matière sèche foliaire observée sur les traitements $\mathrm{T}_{3} \mathrm{C}$ et $\mathrm{T}_{7} \mathrm{C}$ lors du $2^{\mathrm{e}}$ prélèvement est du même ordre de grandeur que les variations de stocks d'amidon mesurées dans des feuilles de tomate soumises à un enrichissement en $\mathrm{CO}_{2}$ (Yelle et al, 1989). TOMGRO ne simule pas ce comportement. Dans le modèle, chaque organe réalise au plus sa croissance potentielle et, si l'offre en assimilats est supérieure à la demande, le surplus est perdu : aucune accumulation d'assimilats au niveau des limbes foliaires n'est possible. Dans le cas de l'enrichissement en $\mathrm{CO}_{2}$, la croissance en surface des feuilles est simulée correctement, mais la surface spécifique (SLA) calculée par le modèle est fausse. L'introduction d'un compartiment de mise en réserve des assimilats permettrait leur accumulation tant que la compétition est faible, puis leur utilisation lorsque l'offre devient limitante.

La demande des tiges a également été ajustée de façon à bien simuler la croissance mesurée. Le rapport constant tige/feuille de 0,49 utilisé dans le modèle initial conduit à une forte surestimation des données expérimentales. Dans le modèle TOMGRO, les variations de $S L A$ se répercutent de la même façon sur la croissance des feuilles et des tiges. Les résultats montrent que les dynamiques de croissance d'une feuille et de la portion de tige correspondante sont différentes et qu'elle sont variables au cours du développement de la plante. Des mesures de la croissance potentielle de la tige et, de la même façon, des racines permettraient de calculer leur croissance de manière indépendante.

Pour les plantes taillées à 3 fleurs par inflorescence, la sous-estimation de la croissance totale s'est répercutée uniquement sur la croissance des fruits. Pour ces 2 traitements, soit la demande totale des fruits est sous-estimée par le modèle, soit l'équilibre de répartition des assimilats calculé par le modèle est faux. La force de puits des différents organes est par définition difficile à mesurer. II est clair cependant que la demande des appareils reproducteur et végétatif est un caractère génétique (Hewitt et al, 1982) et que ces paramètres doivent être évalués pour différents génotypes. Les effets du $\mathrm{CO}_{2}$ et de la température sur la demande des fruits sont peu connus actuellement. Ils sera nécessaire de me- surer ces effets pour élargir le domaine de validité du modèle. L'enrichissement en $\mathrm{CO}_{2}$ augmente l'accumulation de matière sèche dans les fruits (Ho et Hewitt 1986; Tripp et al, 1991). Un effet de la teneur en $\mathrm{CO}_{2}$ sur la force des puits pourrait expliquer la sous-estimation de la demande des fruits pour le traitement $\mathrm{T}_{3} \mathrm{C}$, mais it n'explique pas la sous-estimation du traitement $\mathrm{T}_{3}$. Par contre, l'augmentation de la fraction de matière sèche attribuée par le modèle aux parties végétatives est liée au mode de calcul retenu pour la demande en assimilats des feuilles. La croissance potentielle en surface des feuilles n'est pas affectée par la teneur en $\mathrm{CO}_{2}$ de l'atmosphère, mais elle est convertie en une croissance potentielle en masse à travers une surface spécifique qui diminue sous enrichissement en $\mathrm{CO}_{2}$. L'affectation par le modèle d'une moins grande quantité de matière sèche aux inflorescence à 3 fruits vient du fait que la croissance potentielle du fruit est indépendante de la taille de l'inflorescence et de la position du fruit sur l'inflorescence. Si les fruits sont moins nombreux, leur demande par rapport aux puits végétatifs diminue et le modèle leur affecte une proportion moindre de l'offre en assimilats. Cela peut également être lié à l'hypothèse selon laquelle tous les fruits d'une même inflorescence ont la même croissance potentielle. Or les fruits proximaux auraient une force de puits supérieure aux fruits distaux (Bangerth et Ho, 1984). L'utilisation d'une même croissance potentielle pour l'ensemble des fruits, mais estimée à partir de mesures réalisées sur un fruit proximal, conduit le modèle à surestimer la croissance des fruits sur une plante à 7 fruits par inflorescence.

L'hypothèse d'une corrélation entre la nouaison et la compétition pour les assimilats a déjà été admise par de nombreux auteurs (Picken, 1984; Atherton et Harris, 1986; Wolf et Rudich, 1988). Lorsque le rapport offre/demande en assimilats est bien simulé (taille à 7 fleurs), les résultats montrent que la fonction de nouaison du modèle permet de prévoir avec précision la nouaison. Cependant l'unicité de cette fonction, pour différents niveaux d'activité des sources et des puits d'assimilats, n'a pas été démontrée.

D'une façon générale, il conviendrait de vérifier que le schéma de répartition de la matière sèche de TOMGRO est également respecté dans des conditions d'offre limitante ou si, dans ce cas, certains organes deviennent prioritaires. Chez le pois, le schéma de répartition des assimilats ne semble pas affecté par le niveau 
d'offre total (Jeuffroy, 1991). Chez la tomate, lorsque le pool d'assimilats disponible devient limitant, les puits les plus forts reçoivent une proportion d'assimilats accrue, pénalisant encore plus les puits faibles (Kinet, 1977). Si tel est le cas, il devient donc nécessaire d'introduire des facteurs de priorité dans le modèle et de différencier chaque organe en fonction de sa position sur la plante. Dans le modèle TOMGRO, cette distinction n'est pas possible avec une représentation de la plante en cohortes d'organes différenciés uniquement par leur âge physiologique.

L'objectif des quatre traitements appliqués était de croiser 2 niveaux d'offre (enrichissement en $\mathrm{CO}_{2}$ ) avec 2 niveaux de demande en assimilats (taille des inflorescences) et de tester les hypothèses du modèle dans ces conditions. En pratique, l'enrichissement en $\mathrm{CO}_{2}$ n'a pas permis d'augmenter suffisamment le niveau de l'offre pour observer des différences importantes entre les traitements. La confrontation de la version originale de TOMGRO avec ces données expérimentales permet de souligner le caractère variétal de certains paramètres (sous-modèles de photosynthèse brute et de force des puits). En particulier, la force de puits peut varier fortement entre une variété de tomate à petits fruits et une variété à gros fruits. Le schéma de répartition de la matière sèche en fonction de la force relative des différents puits doit prendre en compte l'existence vraisemblable de priorités ou de hiérarchies au sein de chaque inflorescence. Pour cela, on doit passer à une description de la croissance individuelle de chaque fruit. En revanche, le déterminisme de la nouaison par le rapport offre/demande en assimilats est confirmé expérimentalement. Enfin, l'introduction d'un compartiment d'assimilats mettant en réserve les excédents éventuels de l'offre en assimilats est souhaitable, d'une part, pour simuler correctement les augmentations de matière sèche foliaire sous enrichissement en $\mathrm{CO}_{2}$ et, d'autre part, pour gérer une masse de carbone excédentaire éliminée sans justification par le modèle. Sous ces conditions, on disposera avec le modèle TOMGRO d'un bon outil de prévision des rendements de la tomate, susceptible d'être utilisé pour la définition de stratégies optimales du climat des serres.

\section{REMERCIEMENTS}

Ce travail a été réalisé grâce au support financier de I'APREL (Association provençale de recherche et d'expérimentation légumière). Les auteurs remercient B Brunel pour son appui technique, ainsi que $C$ Orlando pour sa participation aux mesures biologiques.

\section{RÉFÉRENCES}

Acock B, Charles-Edwards DA, Fitter DJ, Hand DW, Ludwig LJ, Warren-Wilson J, Withers AC (1978) The contribution of leaves from different levels within a tomato crop to canopy net photosynthesis: an experimental examination of two canopy models. $J$ Exp Bot 29, 815-827

Atherton JG, Harris GP (1986) Flowering. In : The tomato crop (Atherton JG, Rudich J, eds) Chapman and Hall, London, 167-200

Baille A, Boulard T, Gary C (1990) Les critères d'optimisation dans la gestion du climat et de la production sous serre. CR Acad Agric Fr 5, 25-36

Bangerth F, Ho LC (1984) Fruit position and fruit set sequence in a truss as factors determining final size of tomato fruits. Ann Bot 53, 315-319

Bonnemain JL (1966) Sur les modalités de la distribution des assimilats chez la tomate et sur ses mécanismes. C R Acad Paris 262, 1106-1109

Fisher KJ (1975). Effect of the amount and position of leaf tissue on the yield of single-truss tomatoes. $\mathrm{Sci}$ Hortic 3, 303-308

Gary C (1988) Un modèle simple de simulation des relations microclimat-bilan carboné chez la tomate en phase végétative. agronomie $8,685-692$

Gary C, Jones JW, Longuenesse JJ (1993) Daily changes in specific leaf area: the role of the leaf assimilate pool. Acta Hortic 328, 205-210

Heuvelink E, Marcelis LFM (1989) Dry matter distribution in tomato and cucumber. Acta Hortic 260, 149157

Hewitt JD, Dinar M, Stevens MA (1982) Sink strength of fruits of two tomato genotypes differing in total fruit solids content. J Am Soc Hortic Sci 107, 896-900

Ho LC, Shaw AF, Hammond JBW, Burton KS (1983) Source-sink relationships and carbon metabolism in tomato leaves $1 .{ }^{14} \mathrm{C}$ assimilates compartmentation. Ann Bot 52, 365-372

Ho LC, Hewitt JD (1986) Fruit development. In: The tomato crop (Atherton JG, Rudich J, eds) Chapman and Hall, London, 201-240

Jeuffroy MH (1991) Rôle de la vitesse de croissance, de la répartition des assimilats et de la nutrition azotée, dans l'élaboration du nombre de graines du pois protéagineux de printemps. Thèse de Doctorat, univ Paris-Sud centre d'Orsay, $208 p$

Jones JW, Dayan E, Allen LH, Van Keulen H, Challa $H$ (1991) A dynamic tomato growth and yield model (TOMGRO). Trans ASAE 34, 663-672

Kano A, Van Bavel CHM (1988) Design and test of a simulation model of tomato growth and yield in a greenhouse. J Jpn Soc Hortic Sci 56, 408-416 
Kinet JM (1977) Effect of defoliation and growth substances on the development of the inflorescence in tomato. Sci Hortic 6, 27-35

Maisonneuve B, Philouze J (1982) Action des basses températures nocturnes sur une collection variétale de tomate (Lycopersicon esculentum Mill). I. Étude de la production de fruits et de la valeur fécondante du pollen. agronomie 2, 443-452

Picken AJF (1984) A review of pollination and fruit set in the tomato. J Hortic Sci 59, 1-13

Spitters CJT, Van Keulen $H$, Van Kraalingen DWG (1989) A simple and universal crop growth simulator: SUCROS 87. In: Simulation and system management in crop protection (Rabbinge R, Ward SA, van Laar HH, eds), PUDOC, Wageningen, 147-181

Tchamitchian M, Longuenesse JJ (1991) Phosynthèse d'une culture en rangs de tomates sous serre. Mo- délisation analytique et cartographie de l'activité du feuillage. agronomie 11, 17-26

Tchamitchian M, Longuenesse JJ (1992) A photosynthesis model for greenhouse row crops. Acta hortic 303, 137-144

Tripp KE, Peet MM, Mason Pharr D, Willits DH, Nelson PV (1991) $\mathrm{CO}_{2}$ - enhanced yield and foliar deformation among tomato genotypes in elevated $\mathrm{CO}_{2}$ environments. Plant Physiol 96, 713-719

Wolf S, Rudich J (1988) The growth rates of fruits on different parts of the tomato plant and the effect of water stress on dry weight accumulation. Sci Hortic 34, 1-11

Yelle S, Beeson RC, Trudel MJ, Gosselin A (1989). Acclimation of two tomato species to high atmospheric $\mathrm{CO}_{2}$. Plant Physiol 90, 1465-1472 